\title{
Europa e Iberoamérica: entre la integra- ción y el soberanismo
}

\author{
Europe and Ibero-America: between integration \\ and soveringty
}

Jorge Argüello ${ }^{1}$

Resumen: El mundo atraviesa un momento de grandes transformaciones que llevan a una sensación generalizada de incertidumbre; parece que la realidad internacional se resiste a todos los modelos que antes la explicaban. Este escenario ha afectado y sigue afectando los procesos de integración de Europa e Iberoamérica, tanto dentro de cada región como entre ellas. El presente artículo se divide en tres secciones: primero se aborda la integración como dinámica y como proyecto; luego se desarrollan los procesos de integración en Iberoamérica y Europa; y, finalmente, se discuten las problemáticas globales centrales que influyen sobre sendas regiones.

Palabras claves: Integracion; Europa; Iberoamerica

Abstract: The world is going through a time of great change leading to a general feeling of uncertainty. It seems that the international situation is resisting all the models that used to explain it. Such scenario has affected the European and Ibero-American integration processes, both within and between the regions, and continues to do so. This article consists of three sections: the first one tackles integration as dynamic and as a project, the second one develops the integration processes in Europe and Ibero-America, and the last one discusses the central global issues which have an impact on various regions.

Keywords: Integration; Europe; Iberoamerica

DOI: https://doi.org/10.24215/23142766e064

${ }^{1}$ Presidente de Fundación Embajada Abierta, exembajador ante Naciones Unidas, Estados Unidos y Portugal. Artículo sobre la base de la ponencia presentada en el XIV COLOQUIO INTERNACIONAL - LA INTEGRACIÓN IBEROAMERICANA Y EUROPEA: “UNA VISIÓN DESDE LAS RELACIONES INTERNACIONALES Y LOS DERECHOS HUMANOS EN AMÉRICA Y EUROPA", 23 de abril, La Plata. 


\section{Introducción: sobre la integración}

Para entender qué momento está atravesando la integración iberoamericana y europea, conviene detenerse en aquello que está en juego cuando hablamos de integración. La definición más reconocida dentro de la Teoría de la Integración (Haas, 1958) reza lo siguiente:

"La integración es el proceso en el cual actores políticos en muchos y diversos contextos nacionales optan por reorientar sus lealtades, sus expectativas y sus actividades políticas hacia un nuevo centro; el cual a través de sus instituciones posee o demanda jurisdicción sobre Estados-nación preexistentes".

De esta fórmula hay varios puntos importantes que podemos recordar. El primero es que nunca hay que olvidar que la integración tiene un componente político. En tanto proceso que altera los órdenes establecidos, la integración es uno de los grandes cambios que implica la movilización de voluntades, el manejo del conflicto y las relaciones de poder. Para cualquier caso que estudiemos, encontraremos tensiones en los movimientos que la integración comprende.

El segundo es que la integración no sólo opera en el plano político, sino también es un proceso social. Como la definición lo indica, las lealtades y expectativas de poblaciones enteras son moldeadas ya sea con o en contra del tren de la integración. El tercero, a veces el más olvidado en el día a día, es que los procesos de integración producen resultados institucionales. Regiones que se integran dan vida a instituciones que, con el tiempo, pueden llegar a cobrar dinámicas propias: la integración se vuelve más que la suma de las partes.

El cuarto punto, y de mayor trascendencia, es que la integración trabaja por encima del plano estatal. La idea de la integración, en clave histórica, es extremadamente novedosa por su aspiración de generar relaciones de autoridad y pertenencia más allá del nivel de los Estados-nación. En su vertiente más ambiciosa, la propuesta integracionista afirma que el siglo XXI será el siglo de las regiones: grupos de Estado que atravesarán lógicas de interdependencia como nunca hasta el momento.

Este último punto se comprende mejor si se recuerda que el actor protagónico del sistema internacional durante los últimos siglos ha sido el Estado-nación. Como piedra angular, configuró todo un vocabulario: las nociones de soberanía, igualdad jurídica entre Estados, la no injerencia en los asuntos internos de otros Estados, las cláusulas de nación más favorecida en el plano comercial, entre otras.

Hoy, principalmente producto de las fuerzas de la globalización de las últimas décadas, el Estado se encuentra interpelado "desde arriba" por los organismos y regímenes internacionales; "desde los costados", por actores no estatales, como el crimen transnacional organizado, el terrorismo y las grandes multinacionales; y "desde abajo", por una activa y cada vez más demandante sociedad civil organizada (Kobrin, 1997). El proceso de integración opera en este contexto.

En este sentido, en el mundo hoy tenemos tres certezas respecto de la relación entre la globalización y el Estado-nación. En primer lugar, las visiones inocentes que veían la globalización como una bendición que resolvería todos los males ya no se sostienen. En segundo lugar, por el contrario, existe un consenso acerca de la desigualdad en los ingresos y riquezas globales, y los problemas que ella genera. En tercer lugar, se visibiliza el imperativo de generar nuevas estructuras de gobernanza global que permitan coordinar esfuerzos. Un 
ejemplo que yo he trabajado con asiduidad es el del G20 como espacio de resolución proactiva de la crisis de 2008-2009 y durante toda la década pasada (Argüello, 2018).

En efecto, en el mundo de hoy este es el gran clivaje: la disputa entre soberanistas y globalistas. Mientras que los primeros quieren retener los esquemas tradicionales y son la principal fuerza de inercia política, los segundos ven al cambio como inevitable y deseable, y los encontramos como los principales promotores de la transición.

En los últimos tiempos, lo que estamos presenciando es más un diálogo de sordos que una discusión racional. Los fanáticos de la globalización que pregonan la integración internacional no prestan atención a los detractores: los tildan de proteccionistas hasta la médula, y los acusan de no entender el principio de las ventajas comparativas ni las complejidades de las leyes y la institucionalidad del intercambio comercial. Por otra parte, los críticos de la globalización acusan a los economistas y a los especialistas por su visión poco amplia y tecnocrática: ellos argumentan que los globalistas se encuentran demasiado ensimismados en sus modelos complejos y que no tienen una buena percepción de cómo gira el mundo en verdad. Veamos ahora cómo se ve esto en los casos europeo e iberoamericano.

\section{Integración reciente en Europa e Iberoamérica.}

Ambas regiones presentan similitudes y diferencias en sus integraciones recientes. Para cada región debemos analizar qué proyectos están en coexistencia, qué identidad definen, qué nivel de integración plantean, y en qué contexto se desarrollaron.

\section{iv. Europa}

En el caso europeo, lo que puede apreciarse es que hay una concepción dominante en el proceso de integración: la de la Unión Europea. Esto es importante, pues si bien hay otros organismos o regímenes para el caso europeo -como por ejemplo la Asociación Europea de Libre Comercio-, el proyecto con centro en Bruselas es por lejos el preponderante.

La identidad para esta región desde el inicio del proceso de integración estuvo clara: "europea". Desde la Declaración Schuman en 1950 hasta la fecha, la idea ha sido construir una integración con el objetivo de lograr una "Europa europea". Esta identidad europea no sería incompatible con la local ni nacional de cada ciudadano europeo; por el contrario, se concibe como una pertenencia por capas superpuestas, como indicaba Ortega y Gasset (Beneyto Pérez, 2005). Las reflexiones acerca de la esencia de esta identidad europea giran desde el humanismo, pasando por la tradición judeocristiana, el paradigma científico, el derecho y la filosofía occidental. El artículo 2 del Tratado de la Unión Europea refleja estas concepciones, expresadas en el vocabulario de los Derechos Humanos, la democracia, el Estado de Derecho, la dignidad humana y las libertades fundamentales.

¿Qué extensión geográfica implica esta identidad europea? En plena Guerra Fría, el general De Gaulle concebía una Europa unida "desde el Atlántico hasta los Urales". La identidad regional coincidiría, bajo esta lupa, con la continental. A lo largo de sus siete oleadas de ampliación, de 6 a 28 miembros -si todavía contamos al Reino Unido-, la Unión Europea incorporaba miembros de todos los rincones del continente. Los límites parecen ser en buena medida claros, puesto que los países de la costa sur del Mediterráneo han sido explícitamente rechazados. 
Sin embargo, dos grandes interrogantes se plantean en este aspecto. El primero es la cuestión turca. Por un lado, desde hace más de 50 años que Ankara mantiene con el proyecto de integración negociaciones de acceso e incluso ha llegado a acoplarse en algunos foros u obtener algún estatus de adhesión. Por el otro, la posibilidad de una incorporación turca siempre despierta ciertas reservas: en primer lugar, las diferencias culturales y religiosas. Sumado a ello, Turquía -de entrar- se convertiría en el país más poblado del bloque, superando a nada menos que Alemania. Tercero, una entrada turca implicaría una Unión Europea que llegará hasta Anatolia y que pasará a limitar directamente con Siria, Irán e Irak. Cuarto, en la actualidad las relaciones con Bruselas están en un punto bastante poco dinámico, por los giros importantes que está dando el Presidente Erdogan en materia de política exterior e interior.

El segundo interrogante es el vecino ruso. A pesar de las no menores coincidencias culturales y religiosas, Moscú siempre ha jugado un papel ambivalente. Incluso en su autoimagen actual, con el gobierno de Putin, Rusia titubea a la hora de aceptar definirse como un país "europeo". Durante la Guerra Fría, la Unión Soviética era la última frontera y sinónimo de confrontación. Con la disolución de la URSS, el espacio del "socialismo real" tomó dos caminos: a la fecha, todos los países satélites del Pacto de Varsovia están en la Unión Europea. Sin embargo, no todos los países que componían la URSS se incorporaron al proyecto europeo. Mientras que los países bálticos se sumaron, una incorporación de Ucrania hoy parece estar más lejos que nunca. De manera similar al caso turco, la incorporación rusa nos daría una Unión Europea que pasaría a limitar con Corea del Norte, la propia China, y se acercaría a tan solo 85 kilómetros de Estados Unidos. Pues, de otro modo, ¿cómo podría aceptar Rusia que sólo una parte de la federación se sumara a la UE? ¿Sería acaso posible en términos prácticos?

La tercera pregunta, referida al nivel de integración, en el caso de la Unión Europea tiene una respuesta excepcional. Por su amplitud y profundidad, la propuesta europea ha servido de paradigma para que otras regiones hicieran lo propio. Gracias a sus múltiples capítulos, la Unión Europea sigue siendo el significado común para cualquiera que enuncie el término "integración" (Staab, 2013).

Las cuatro libertades de las que goza el espacio europeo (bienes, servicios, personas, capital) cementan una sólida interacción entre sus miembros. De manera similar, la unión monetaria con 19 miembros en la actualidad constituye otra apuesta fuerte en el plano económico de la integración. Además de estos países, 7 miembros de la UE deben implementarlo a futuro y otros 6 que no son parte de la Unión lo utilizan.

La justicia también es un rasgo saliente del proyecto de integración europeo, con un Tribunal de Justicia que en su labor no sólo ha velado por resolver los conflictos del proceso de convergencia, sino que sistemáticamente ha actuado en pos de mayor integración. El área compleja para el proyecto de integración europeo sigue siendo el de la seguridad común. Recién con el Tratado de Lisboa, hace 10 años, se dio forma sustancial a una Política Exterior y de Seguridad Común (la PESC). Sin embargo, todavía hoy de todos los altos funcionarios, la única cartera que no tiene el rango de Ministerio es la del Alto representante de la Unión para Asuntos Exteriores y Política de Seguridad, en la actualidad a cargo de Federica Mogherini.

Esto nos lleva a la cuarta pregunta, referida al contexto del proceso de integración. El caso europeo fue estructurado directamente por las fuerzas sistémicas de la Guerra Fría: en la década de 1950, en los primeros pasos de la integración, la defensa no estuvo dentro de 
los planes comunitarios. Esto tiene que ver, en gran medida, con la presencia de Estados Unidos como "pacificador externo" y la arquitectura de la OTAN, que les permitió a los europeos en buena medida delegar el área de la defensa, no sin conflictos de por medio. De hecho, con la perdurabilidad del pacto de seguridad hasta nuestros días, los miembros de la Unión Europea son a su vez miembros de la alianza atlántica. Las grandes excepciones siguen siendo Irlanda, Austria y Suecia.

Al mismo tiempo, el proceso de integración fue de la mano con el de la descolonización. Una Europa que se retiraba del mundo luego de 500 años, necesitaba redefinirse y conservar unida su relevancia dentro del plano internacional. La certeza fue que la cooperación y una voluntad coordinada mantendrían a quien supo ser el centro al menos en la mesa chica. Con pocos años de diferencia, la Europa que promulgaba el Tratado de Maastricht (1993) se retiraba de Hong Kong (1997, Reino Unido) y Macao (1999, Portugal).

\section{v. Iberoamérica}

Para el caso iberoamericano, en vez de un proyecto dominante, encontramos por lo menos dieciseis discordantes: la Alianza Bolivariana para los Pueblos de Nuestra América (ALBA), la Alianza del Pacífico, la Asociación de Estados del Caribe, la Asociación Latinoamericana de Integración, la Comunidad Andina, la Comunidad del Caribe (CARICOM), la Comunidad de Estados Latinoamericanos y Caribeños (CELAC), el Mercado Común Centroamericano, el Mercado Común del Sur (MERCOSUR), la Organización de Estados del Caribe Oriental, la Organización del Tratado de Cooperación Amazónica, el Proyecto Mesoamérica, el Sistema de la Integración Centroamericana (SICA), el Sistema Económico Latinoamericano y del Caribe, la Unión de Naciones Suramericanas (UNASUR) y el flamante PROSUR - Foro para el Progreso y el Desarrollo de América Latina.

Todos estos organismos varían ostensiblemente en su composición, límites geográficos y antigüedad. En vez de un gran proyecto mancomunado, Iberoamérica ha encauzado sus esfuerzos supranacionales por distintos caminos. A ello se le suma la tendencia de marchas y contramarchas al interior de cada uno de estos organismos ante los cambios políticos domésticos (Kacowicz, 2008).

La identidad es otro asunto complejo. Lo primero por destacar es que la herencia religiosa, cultural, lingüística y social para poder hablar de una Iberoamérica es innegable. A lo largo del último medio milenio, gran parte del continente americano transitó el proceso de formación de sus principales rasgos actuales. Sin embargo, más allá de esa gran identidad iberoamericana compartida, la integración iberoamericana reflejó fragmentos de ella. Los puntos de corte son varios: regionales, como la sudamericana vs. la latinoamericana; o la centroamericana vs. la caribeña; comerciales, como la atlántica vs. la pacífica; identitarios, como la andina vs. la amazónica vs. la bolivariana.

La extensión iberoamericana sería, en principio, un poco más clara, en vinculación directa con los países de la Península Ibérica, otrora metrópolis: España y Portugal; además de Andorra recientemente.

La tercera pregunta, respecto de los niveles de integración, también se encuentra con la multivocalidad de Iberoamérica. Los distintos organismos oscilan entre la integración comercial, la unión aduanera, la cooperación educativa y tecnológica, la coordinación en defensa común, la colaboración en seguridad interna, la asistencia humanitaria, la mediación política, y la representación parlamentaria supranacional. Esto ofrece un panorama mucho 
más fractal que el europeo, donde un mismo país puede ser miembro de distintos organismos, en distintas condiciones, cada uno con diferente alcance geográfico, agendas particulares, y espacios de negociación diversos.

No obstante esta complejidad, la integración iberoamericana ha seguido su curso sin retrocesos importantes. Ni siquiera las crisis en el continente desbarataron las arquitecturas regionales, como la suspensión de Cuba, los golpes de Estado más recientes en Honduras y Paraguay, las catástrofes naturales o los defaults económicos. Además de los europeos, los iberoamericanos avanzamos sin pausa en las últimas décadas por más integración y sostenemos importantes consensos como el respeto por los derechos humanos, la importancia de los regímenes constitucionales y democráticos, la región como vecindario de paz ausente de grandes conflictos interestatales y la garantía de una zona libre de armas nucleares.

Finalmente, el contexto de integración. Todos los organismos iberoamericanos operan en coexistencia con el paraguas hemisférico de la Organización de los Estados Americanos (OEA), incluso antes de que la integración europea diera sus primeros pasos (1948). Al estar Estados Unidos en el continente americano, juega un rol mayor que el de pacificador externo y colaborador como lo hace para el caso europeo: es miembro pleno del principal organismo continental y sigue, con alta prioridad estratégica, todo suceso relacionado con sus vecinos del sur. La identidad iberoamericana que impulsa una integración en esa clave entra entonces en conflicto directo con los países de mayor extensión del continente: Estados Unidos y Canadá.

Dejando de lado esta cuestión, los distintos hitos de la integración iberoamericana han ido de la mano de mareas políticas y económicas comunes a toda la "Patria Grande". La textura de la integración regional ha ido variando según las oleadas de autocratización y democratización política, de liberalización y proteccionismo económico, de nuevas izquierdas y nuevas derechas. En cada momento, lo importante es que hubo una voluntad política de redefinir a lberoamérica en nuevas visiones de sí misma y sobre todo para relacionarse con el resto del mundo. Igual que los europeos, los iberoamericanos entendemos que podemos llegar más lejos y granjear mejores triunfos si nos sentamos con una voz única en la mesa de negociación; sobre todo si sumamos nuestra condición periférica en el tablero global.

\section{Los procesos de integración de Europa e Iberoamérica hoy.}

Teniendo esta base para el análisis, podemos pasar a abordar los desafíos que enfrentan ambos procesos de integración y el contexto en el cual se están produciendo las transformaciones actuales. Ya subrayamos anteriormente la puja entre los globalistas y los soberanistas. En efecto, ese es el principal parteaguas, pero ¿qué problemáticas globales impactan sobre la integración europea e iberoamericana? Tres de ellas son insoslayables para atender el futuro de la integración: los cambios por encima de los Estados (la esclerosis del multilateralismo y la falta de rumbo en la gobernanza global), los cambios en algunos Estados (Estados Unidos y China) y los cambios dentro de los Estados (la crisis de los sistemas tradicionales y los nuevos liderazgos personalistas).

\section{vi. Esclerosis del multilateralismo y falta de rumbo en la gobernanza global}

El regionalismo es una forma de multilateralismo. Luego de la Segunda Guerra Mun- 
dial, la comunidad internacional sostuvo que para poder lograr avances colectivos eran necesarias instituciones multilaterales. En este marco, todos los organismos que fueron mencionados en esta presentación nacieron, se desarrollaron y se readaptaron.

Hoy en día la cosa ha cambiado: abunda un pesimismo respecto de las instituciones multilaterales. Las críticas señalan su lentitud, su acartonamiento, su condición de fachada de consenso funcional a los más poderosos y su incapacidad de ajuste a los nuevos problemas.

Esto ha llevado a varios países a, o bien retirarse, o bien disminuir sus interacciones dentro de ellos, o a complementarlas con el plano regional. Este último punto, el del regionalismo, ha sido el fenómeno en paralelo a la esclerosis del multilateralismo más notorio en los últimos lustros. Entonces, producto de este problema, el regionalismo sale a la vez fortalecido -porque los países se retiran del plano global para confiar en su vecindario- y debilitado, porque los países prefieren cada vez más tomar medidas unilaterales para maximizar sus beneficios y minimizar sus daños.

De manera similar, el regionalismo ha ido variando entre concepciones más abiertas de regionalismo (como el Mercosur o la CARICOM) y concepciones más cerradas de regionalismo (UNASUR/ALBA). En la actualidad, lo que está ocurriendo con el PROSUR en nuestra región debe interpretarse bajo esta clave. Estamos asistiendo al pasaje de un regionalismo cerrado a uno abierto, más orientado hacia la apertura económica y las relaciones NorteSur.

El multilateralismo es necesario en Europa e Iberoamérica por tres motivos: el primero de ellos es que la naturaleza de los problemas globales demanda como nunca una acción consensuada y eficaz: desde el cambio climático hasta las migraciones, desde el crimen transnacional organizado a la cuestión de la tercera edad. Ejemplo de ello es la administración que Mogherini ha intentado encauzar en la UE para la crisis de refugiados de los últimos años.

El segundo motivo es que, ante un contexto de incertidumbre extrema, las instituciones multilaterales operan en un marco de previsibilidad muy valioso. Ejemplo de ello fue el manejo de la crisis paraguaya en 2012 en el marco del Mercosur.

El tercer motivo reside en que, en un mundo crecientemente desigual, el multilateralismo ofrece una plataforma a los menos poderosos para maximizar sus capacidades y limitar la discrecionalidad de los más poderosos. Ejemplo de ello es la coordinación europea en las sanciones contra Rusia por el conflicto en Crimea y el este de Ucrania desde 2014.

En el contexto anterior, se ha planteado cada vez más la pregunta "¿quién está a cargo?". Existen varias respuestas para esa pregunta: el G8, G20, G2, G-Cero o un mundo Multi-G. Lo cierto es que, si bien la integración no puede reemplazar la gobernanza global, sí puede facilitar la coordinación entre regiones proporcionando los simientos para un mejor manejo de las relaciones internacionales. Ejemplos de ello encontramos en la presencia de la UE en el G20 y la invitación del CARICOM a la última cumbre en Buenos Aires el año pasado.

\section{vii. El atrincheramiento de Estados Unidos y el ascenso de China}

Ambas integraciones no pueden desentenderse de las actitudes de dos jugadores del sistema: Estados Unidos y China. En primer lugar, en materia de política exterior, Trump aboga por reducir la injerencia estadounidense en conflictos internacionales, pero, por el otro lado, quiere mantener el rol de Estados Unidos como garante del orden internacional. En virtud de ello, Trump ha declarado como objetivos el restaurar la paz mundial, eliminar 
al Estado Islámico y contener al Islam radicalizado. A la vez, es difícil conciliar estos objetivos con la búsqueda de una política menos intervencionista, como la que ha abogado en su discurso de campaña America First sobre política exterior, asegurando que en su gobierno Estados Unidos dejaría de someter a su pueblo a las falsas promesas del globalismo.

Este diagnóstico toma mejor forma si consideramos a Trump en el terreno de la política exterior como un jacksoniano, haciendo uso de la clasificación de escuelas de política exterior estadounidense. Su diplomacia de corte expeditivo y lineal, su desconfianza en un gobierno federal demasiado grande y elucubrado, y su recurrencia al poder militar cuando fuere estrictamente necesario, combinadas con su máxima de que el orgullo norteamericano ha sido mancillado, son todas características propias de un líder jacksoniano (Listrani y Zaccato, 2017).

Por un lado, anunciaba la retirada de Estados Unidos del mundo a la vez que criticaba abiertamente a los tradicionales aliados de Washington -como Europa Occidental, Japón y Corea del Sur-, demandándoles una mayor contribución (en dinero y en tropas) al esfuerzo militar que hace Estados Unidos. Por el otro, lanzaba su convicción de que los Estados que entorpecieran su plan de engrandecer a Estados Unidos serían castigados. En otros casos, Trump se ha querido encargar personalmente de conflictos delicados y complejos (Corea del Norte, Venezuela y la frontera con México).

Esto obedece a la táctica típica de Trump: primero, tomar medidas unilaterales, causar daño en sectores clave del otro país, y luego ante las medidas recíprocas entablar una negociación desde una situación relativamente ventajosa. En resumen, las decisiones de Trump sobre casos como el de Siria o Rusia afectan directamente los cálculos de la integración europea. Del mismo modo, el proteccionismo comercial o un endurecimiento de la política migratoria y un muro con México impactarán sobre los países iberoamericanos. Los europeos, además de Estados Unidos, son quienes históricamente han sostenido el orden internacional liberal.

Por otro lado, el ascenso chino será algo fundamental para orientar los procesos de integración en las próximas décadas. En 2017, el gobierno chino anunciaba la entrada formal en una "nueva era" para China. Desde el inicio de la reforma y la apertura del país en el último cuarto del siglo pasado, el Partido Comunista de China persiste en el principio de la emancipación mental y la búsqueda de la verdad en los hechos. Partiendo de la realidad de China, reestableció la estrategia de desarrollo de la modernización del país.

El nivel de vida del pueblo ha alcanzado al de una sociedad modestamente acomodada y China comenzó su grandiosa marcha hacia el objetivo de la tercera etapa de alto nivel de desarrollo. Esto tiene como corolario una trasformación del peso chino en el sistema internacional: ese proceso que persigue objetivos internos está indudablemente vinculado con el éxito de objetivos externos. Para poder seguir avanzando, China se ha visto en la necesidad de proyectarse hacia afuera como un jugador preeminente, intentando no generar demasiadas reservas por parte del resto de los jugadores.

En este marco, destaca la iniciativa de la llamada "Nueva Ruta de la Seda". El proyecto One Belt, One Road (OBOR) de China se conoce como el gran plan nacional a largo plazo para las próximas dos o tres décadas. Este se compone de dos partes: un "Cinturón Económico de la Ruta de la Seda", que se extiende sobre la masa terrestre de Eurasia, y una "Ruta de la Seda Marítima del Siglo XXI", que abandona las costas de China y pasa por el Océano Índico, el Canal de Suez y el Mar Mediterráneo para llegar a Europa. Cabe señalar que hasta ahora China nunca ha definido con precisión las rutas exactas, ni para el cinturón ni para la 
carretera, más bien son concesiones políticas que se van detallando en la misma ejecución del plan.

El eje económico se está desplazando del Atlántico al Pacífico. Europa por tierra e lberoamérica por mar necesitan dar debida cuenta de las implicancias que esto trae. Por un lado, la imposibilidad de quedar fuera del nuevo reparto de cartas, con los riesgos que supone actuar en forma inconexa cada país por separado. Por el otro, la potencialidad de una mayor integración entre Europa e Iberoamérica en un futuro en que el espacio atlántico no se encuentre tanto en el centro de las miradas.

\section{viii. La crisis de los sistemas tradicionales y los nuevos liderazgos personalistas.}

¿Están en problemas los partidos políticos? ¿Son tan influyentes como solían serlo en términos de su capacidad para reclutar y retener miembros, conseguir que sus votantes acudan a las elecciones y mantener la lealtad de los votantes de una elección a otra? Los datos muestran que nuestros países han experimentado una disminución de la influencia de los partidos políticos en términos de disminución de la membresía, la participación de votantes y la estabilidad del sistema de partidos, con implicaciones para la rendición de cuentas democrática, la institucionalización de la competencia política y la calidad de la propia democracia. De gran preocupación, Europa -la región en la que nacieron y se institucionalizaron mejor los partidos políticos- ha mostrado algunos de los indicadores más claros y negativos.

Estamos viendo cómo, en el mundo, la valoración de los partidos políticos tradicionales está en unos niveles inusitadamente bajos. La desconfianza y la desilusión de los ciudadanos respecto de los partidos políticos es ciertamente indudable. Por un lado, buscan cada vez más personas preparadas y calificadas para sacar adelante una sociedad moderna muy compleja. Al mismo tiempo, la sociedad civil está cansada de los casos de corrupción y de la falta de responsabilidad frente a determinadas gestiones públicas. La crisis económica y financiera ya mencionada ha demostrado cómo los controles han fallado y, en última instancia, no se ha actuado con acierto.

Las cosas han cambiado. Ahora los partidos tienen que ser eficientes gestores, informar con transparencia y estar, por encima de todo, "al servicio del ciudadano". Pero en muchos casos esto ha sido imposible: se ha terminado por asociar a los principales referentes políticos como parte de una élite estatal que responde a sus propios intereses de grupo más que a la ciudadanía. Adicionalmente, este distanciamiento de los dirigentes de sus bases también actuó en detrimento de la renovación de los cuadros políticos. Esto tiene un corolario importante en términos de la percepción de los partidos por parte de la sociedad: en vez de agregar las preferencias de la ciudadanía, las ignoran; en vez de ser el principal vehículo de representación, alejan a los individuos de la toma de decisiones.

Por lo tanto, paradójicamente, en una época en la que se demandan estructuras mucho más ágiles y flexibles, el sistema político que los partidos tradicionales habitan se ha tornado lento y rígido. Las consecuencias de este fenómeno son las bajas tasas de afiliación de los partidos políticos tradicionales, la baja capacidad para compeler a sus seguidores a las urnas, la declinante estabilidad de los sistemas de partidos y una menor armonía en las bases sociales de los partidos políticos tradicionales. En Europa, esto se conoce como el "déficit democrático" que se le achaca a la UE. En Iberoamérica, las crisis de los partidos tradicionales y el ascenso de movimientos extrapartidarios, sobre todo en contextos de crisis económica. 
En contraposición, el escenario político europeo e iberoamericano ha abierto paso al ascenso de partidos y candidatos de derecha, localistas, nativistas, euroescépticos y, muchas veces, hasta abiertamente xenófobos y racistas; ha desdibujado el tradicional clivaje liberal/conservador que supo estructurar la puja política desde la segunda posguerra. Estos nuevos candidatos desafían las categorizaciones tradicionales. Muchas veces sus discursos sociopolíticos se asemejan a los de los partidos del ala conservadora y más cercanos a la derecha del espectro ideológico, pero al analizar sus discursos en materia económica, comercial y laboral, encontramos ideas y plataformas más cercanas a algunas corrientes de izquierda, como lo son la defensa de políticas proteccionistas, el foco en la creación de empleo y los incentivos a la industria local, entre otras.

No obstante, la división entre localistas y globalistas tampoco se condice con aquella que distingue entre partidos y candidatos de izquierda y derecha, ya que, hoy en día, existen casos de políticos de izquierda y localistas, de izquierda y globalistas, de derecha y localistas, y de derecha y globalistas. Es por esta razón que el escenario político europeo en la actualidad es percibido como turbulento y confuso, pues los espacios políticos y los códigos de cultura política emergidos de la segunda posguerra han perdido en buena medida vigencia. Más aún, con la elección de Donald Trump como presidente de los Estados Unidos, el Brexit y el relativo éxito de partidos nacionalistas en diversos países europeos, el término populismo parece haber tomado el centro de la escena del análisis político. Es evidente, entonces, que las dimensiones de análisis tradicionales resultan hoy insuficientes.

Menos de treinta años después de que Fukuyama y otros declararan el dominio eterno de la democracia liberal, se manifiesta una "tercera ola de autocratización". La disminución gradual de los atributos del régimen democrático caracteriza la autocratización contemporánea. La ola de autocratización que se está desarrollando afecta principalmente las democracias con reveses graduales bajo una fachada legal.

¿Qué características tiene esta oleada? Existe una novedad estructural importante de la tercera ola de autocratización: afecta principalmente las democracias, mucho más que en las primeras olas. Y esto ocurre mientras que el nivel global de democracia está cerca de un máximo histórico con cerca de $56 \%$ de los estados del mundo en democracia. Podría decirse que la pérdida de rasgos democráticos en regímenes que eran democráticos antes es más importante para el estado de la democracia en el mundo que un mayor deterioro de regímenes ya autocráticos. En tres de cada cuatro casos, estas autocratizaciones son lideradas por los que llegaron al poder legal y típicamente por mecanismos democráticos. Por ejemplo, nadie pondría en discusión la legitimidad de origen de figuras como Jair Bolsonaro en Brasil o Mateo Morawiecki en Polonia. Sin embargo, ante el descuido de sus pares, las situaciones de Daniel Ortega en Nicaragua o Viktor Orbán en Hungría se han tornado preocupantes.

Por fortuna, la autocratización es mucho más lenta que en oleadas anteriores. En la actualidad, la mayoría de los regímenes -incluso las autocracias- tienen alguna forma de elecciones multipartidistas. Los movimientos repentinos e ilegales hacia la autocracia tienden a provocar la oposición nacional e internacional. Los autoritarios contemporáneos han aprendido su lección de las oleadas pasadas y, por lo tanto, ahora proceden de una manera mucho más lenta y menos notoria que sus predecesores históricos. Así, mientras la democracia se ha visto sin duda amenazada, su poder normativo todavía parece forzar a los aspirantes autócratas a jugar maniobras de engaño. 
En consecuencia, los Estados afectados por la tercera ola de autocratización siguen siendo mucho más democráticos que sus primos históricos. Por un lado, esto da esperanzas de que la actual ola de autocratización pueda ser más leve que la primera y segunda ola. Debido a que la autocratización es más gradual, los actores democráticos pueden seguir siendo lo suficientemente fuertes como para movilizar la resistencia.

¿Qué pasa con las democracias que sobreviven sin deslizarse hacia el autoritarismo? Por este otro lado, en muchas democracias se habla cada vez más del ya mencionado "déficit democrático": los procesos e instituciones gubernamentales son tan complejos que la ciudadanía en general no los comprende y, por consiguiente, no puede expresar sus preferencias. Además, en muchos casos los cargos y las decisiones no gozan de la más mínima consulta popular. Esto se ha traducido en un rechazo a las instituciones y bajas tasas de participación electoral, en un marco de creciente anomia por la falta de expectativas de que las cosas cambien para mejor.

En suma, el difícil acceso a la información inicial se termina realimentando y produciendo más incertidumbre cada vez. Este es el principal dilema que, por ejemplo, hoy atraviesa la Unión Europea y que motivó en parte el Brexit. Iberoamérica necesita también coordinar respuestas regionales serias y cuidadosas frente a crisis importantes como las ya mencionadas o la de Venezuela.

\section{Conclusión}

La integración es positiva y deseable para los países Iberoamérica y Europa. Los enormes cambios que estamos viviendo desafían todos los modelos, pronósticos y sistemas de creencias previos. Los costos de una parálisis regional o la fragmentación, si retrocedemos, serían demasiado altos. A nivel regional y transatlántico, sobre los abundantes valores compartidos y experiencias previas, necesitamos problematizar, diseñar y consensuar iniciativas que permitan a nuestras queridas regiones navegar en aguas más inciertas que nunca.

\section{Bibliografía}

Argüello, Jorge (2018). ¿Quién gobierna el mundo? El rol del G20 en el nuevo orden mundial. Buenos Aires: Capital Intelectual.

Beneyto Pérez, José María (2005). “Europa como paradigma de la filosofía de la integración de Ortega y Gasset”, en Revista de Estudios Europeos, 40, pp. 91-102.

Cantir, Cristian y Schrodt, Philip. (2010). Neomedievalism in the Twenty-first Century: Warlords, Gangs and Transnational Militarized Actors as a Challenge to Sovereign Preeminence. Pennsylvania State University Press.

Castells, Manuel (2001). La era de la información, España, Alianza, v. III, p. 401.

Haas, Ernst (1958). The Uniting of Europe. Stanford: Stanford University Press.

Holsti, Kal (1985). The Dividing Discipline: Hegemony and Diversity in International Theory. Boston: Allen and Unwin. 
Kacowicz, Arie (2008). América Latina en el mundo: globalización, regionalización y fragmentación. Revista Nueva Sociedad, Vol. 214, marzo-abril.

Kagan, Donald (1995). On the Origins of War and the Preservation of Peace. New York: Doubleday.

Keohane, Robert y Nye, Joseph (2000). Globalization: What's new? What's not? (And so what?). Foreign Policy, Vol. 118, Spring.

Kobrin, Stepjen (1997). Back to the Future: Neomedievalism and the Postmodern Digital World Economy.

Listrani, Tomás y Zaccato, Carolina (2017). "El revoltoso jacksoniano: primeros pasos de política exterior de la administración Trump”, en Ágora Internacional, junio 2017.

Rodrik, Dani (2012). The Globalization Paradox: Why Global Markets, States and Democracy Can't Coexist. OUP, Oxford.

Russell, Roberto (1998). La globalización: situación y proceso. Revista Ciclos, Año VIII, Vol. VIII, Número especial 14-15, Primer Semestre.

Staab, Andreas (2013). The European Union Explained: Institutions, Actors, Global Impact. Bloomington: Indiana University Press.

Waltz, Kenneth (1999). Globalization and Governance. PS Online, December.

Wendt, Alexander (1992). Anarchy is What the States Make of It: The Social Construction of Power Politics, en International Organization, Vol. 46, No. 2 